\title{
A large rectangle delays the perception of a separate small rectangle
}

\author{
DONALD L. KING \\ Howard University, Washington, D.C.
}

\begin{abstract}
Deciding whether two objects, rather than one, are present takes longer for large-small and small-large pairs of rectangles than for large-large and small-small pairs of rectangles. This large-small slowdown was eliminated when the large rectangle was slightly modified, when the large and small rectangles were contiguous, or when the task was to identify the large rectangle. However, it did occur when the task was to identify the small rectangle. These results suggest that the large rectangle delayed the perception of the small rectangle. Codes for features did not cause this delay. Instead, the evidence indicates that each rectangle evoked its own superordinate code, and that the large-rectangle superordinate code produced the gestalt of a large rectangle and also inhibited the small-rectangle superordinate code, thereby delaying the perception of the gestalt of a small rectangle. Thus, superordinate codes may also be the direct cause of other gestalts and their associated perceptual outcomes.
\end{abstract}

A gestalt (perceived unit, experienced entity) is frequently associated with other perceptual outcomes. Thus, classification and Stroop interference disappear when the target parts belong to different gestalts than the irrelevant parts (Garner \& Felfoldy, 1970; Kahneman \& Henik, 1981). Identification of multiple targets is better when they belong to the same gestalt than when they belong to different gestalts (Fryklund, 1975; Pollatsek \& Digman, 1977). Identification of a single target is poorer when it is a member of a gestalt than when it forms its own singleton gestalt (Banks \& Prinzmetal, 1976; Prinzmetal \& Banks, 1977). Similarly, illusory conjunctions between parts are more frequent when they are members of the same gestalt (Prinzmetal, 1981; Prinzmetal \& Keysar, 1989). The time to search for a target increases with the number of gestalts that the noise items produce (Bundesen \& Pedersen, 1983; Ceraso, Powers, \& Moncrief, 1988). Strong single gestalts are associated with fast "same" and slow "different" responses (King, 1988c).

In addition, evidence suggests the principle that (1) a gestalt is consistently associated with an increase in perceived similarity (i.e., assimilation) between parts that be-

This research was supported by the Howard University Faculty Research Support Grant Program and by National Institute of Mental Health ADAMHA-MARC Grant T-34-MH16580. The contributions of the reviewers and editor are appreciated. The discussion of this research with Howard Egeth, Steven Yantis, and others at Johns Hopkins was also helpful. Argie Allen, Carol Chang, Dawn Cooper, Charles Johnson, Debra Johnson, and Rodney Sadler provided assistance with data collection and analysis. The regular-rectangles and circles experiments were reported at the Eastern Psychological Association meeting in Arlington, VA, 1987. The identification, large(modified)-rectangle, and lines experiments were reported at the Psychonomic Society meeting in Seattle, WA, 1987. Please send correspondence concerning this article to Donald L. King, Department of Psychology, Howard University, 525 Bryant St., N.W., Washington, DC 20059. long to it, and (2) two gestalts are frequently associated with a decrease in perceived similarity (i.e., contrast) between parts that belong to different gestalts (King, 1988a, $1988 \mathrm{~b}$ ). Furthermore, this principle holds across areas as diverse as the anchor-range effect (Gravetter \& Lockhead, 1973; Pollack, 1952), the resolution of perceptual differences (Welch, 1978), and the aftereffect obtained by presenting one object and then a second similar object (Gibson, 1933). With figural stimuli, an extreme (anchor) object strengthens the gestalt established by two other objects and also increases the perceived similarity of the other objects (King \& Atef-vahid, 1986; Lockhead, 1988). For example, in the research of Gaylord (1984) that Lockhead describes, a spatially extreme third dot made two other dots group and also made them appear closer together (i.e., in more similar spatial positions). Therefore, in the traditional absolute identification task, an extreme object may make other objects group and may also increase their perceived similarity, hence the difficulty in discriminating among them.

As to perceptual differences, two parts that result in different perceptions when they occur alone nevertheless belong to the same gestalt when they occur together (Gogel, 1977, p. 132; Welch \& Warren, 1980), provided that the difference in the two perceptions is not too great. In addition, the perceptual change that ensues when these parts occur together can be considered as assimilation. For example, proprioception alone may indicate one spatial direction, whereas vision alone may indicate a second spatial direction (due to the use of a prism). Although these two parts differ in direction, when they occur together the arm is apprehended as a single gestalt. Also, the perceived similarity of these two parts increases (assimilation), according to postprism tests.

Whereas two simultaneously present objects result in one gestalt and assimilation, a temporal separation be- 
tween such objects results in each object producing its own gestalt and an aftereffect-that is, contrast (Brigell \& Uhlarik, 1979; Ikeda \& Obonai, 1955, cited in Sagara \& Oyama, 1957; Piaget \& Lambercier, 1944, cited in Pollack, 1964). For example, whereas two simultaneously present horizontal lines of a similar length result in the single gestalt of a horizontal bar and assimilation, a temporal separation between these lines results in each line producing its own gestalt and contrast.

\section{Superordinate-Is-Causal Theory}

A superordinate-is-causal theory accounts for associations between gestalts and other perceptual outcomes as follows. Component stimuli (features) activate a large number of neural detectors and hence generate a large number of neural codes, which somehow combine to produce one or two or perhaps a limited number of additional superordinate codes. Furthermore, a superordinate code is the direct (immediate) cause of a both a gestalt and the perceptual outcomes associated with this gestalt; similarly, two superordinate codes are the direct causes of two gestalts and the perceptual outcomes associated with these gestalts.

Thus, superordinate-is-causal theory attributes assimilation among the parts of a gestalt to an action of the superordinate code that produces this gestalt: The superordinate code causes (in an unknown way) an increase in this assimilation. Likewise, it attributes contrast between the parts of two different gestalts to an interaction between the superordinate codes for these gestalts. So the aftereffect need not be due solely to neural fatigue or photopigment depletion.

Superordinate-is-causal theory resembles Lockhead's (1972) theory of perceptual discriminations and the identification of the parts of stimuli: Both superordinate codes and blobs may directly (immediately) influence perception. Furthermore, the number of such superordinate events that occur-one or two-is pivotal. The opposing and more popular theory is that features result in a large number of neural codes and that at least one of these codes directly influences perception.

\section{The Present Research}

The purpose of the present research was to test superordinate-is-causal theory. The operating assumption, which the results ultimately supported, was that a large rectangle evokes a superordinate code and that a spatially separate small rectangle evokes a second superordinate code; that the first superordinate code produces the gestalt of a large rectangle and that the second superordinate code produces the gestalt of a small rectangle; and that the large-rectangle superordinate code also inhibits the smallrectangle superordinate code, thereby delaying the perception of the small-rectangle gestalt.

In the initial experiment, the task was to identify either the large or the small rectangle. In the subsequent experiments, the task was to respond either when one object was present or when two objects were present. The identi-

fication experiment and one of the 1-versus- 2 experiments employed spatially separate large and small rectangles and obtained evidence that the large rectangle delayed the perception of the small rectangle. In two other experiments, one or both rectangles were altered to eliminate featural and additional explanations of the presumed delay.

\section{THE IDENTIFICATION EXPERIMENT}

As shown in Figure 1, the two-object stimuli used consisted of large-large, large-small, small-large, and smallsmall pairs of open rectangles that were either near or far apart. The one-object stimuli were the individual objects of the two-object stimuli.

Phenomenally, each rectangle is perceived as a single gestalt: The four lines of each rectangle and the white space that is internal to these lines are perceived as a unit, rather than as independent. According to the operating assumption, the large-rectangle superordinate code inhibits the small-rectangle superordinate code, thereby delaying the perception of the small-rectangle gestalt. Therefore, the prediction was that the large-small and small-large stimuli would slow down the identification of the small rectangle but not that of the large rectangle. This asymmetrical slowdown prediction is consistent with evidence that large objects are usually more quickly identified than are small objects (Eriksen \& Schultz, 1979; Grice, Canham, \& Boroughs, 1983; Navon, 1977; Schultz \& Eriksen, 1978).

NEAR

\section{LARGE -}

LARGE

SMALL SMALL

LARGE SMALL

SMALL IARGE
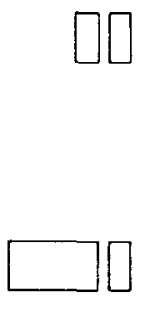
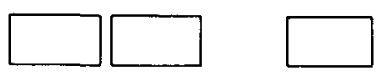

FAR
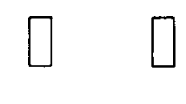

Figure 1. The two-object stimuli for the identification and regularrectangles experiments. 
Feature theory would attribute the predicted asymmetrical slowdown to features of the large rectangle. In order to minimize the difference in features between the large and small rectangles, the rectangles were open. In addition, they were positioned so that the features of the large and small rectangles would be identical near the center of the total stimulus. With this arrangement, differences in features between the large and small rectangles would have to be influential over a relatively large distance. Furthermore, the distances between rectangles were far as well as near in order to increase the extent over which these differences in features would have to be influential. Explanations of the predicted asymmetrical slowdown that are not based on the large rectangle's features are also considered below.

\section{Method}

Subjects. Eleven subjects were instructed to press a telegraph key as quickly as possible without making errors whenever a large rectangle-either one or two-occurred. Another 11 subjects were instructed to respond in this manner whenever a small rectangle occurred. The subjects were students from the introductory psychology course at Howard University.

Stimuli. A Gerbrands G1132 three-channel tachistoscope was used. The large and small rectangles were $20 \mathrm{~mm}\left(1.36^{\circ}\right)$ and $5 \mathrm{~mm}$ $\left(0.34^{\circ}\right)$ wide, respectively, and both were $11 \mathrm{~mm}\left(0.75^{\circ}\right)$ high. The near and far edge-to-edge separations between the two rectangles were $2 \mathrm{~mm}\left(0.14^{\circ}\right)$ and $22 \mathrm{~mm}\left(1.50^{\circ}\right)$, respectively. The midway distance between the two rectangles was at the center of the tachistoscope card. Single rectangles occurred in the same spatial positions as the double rectangles.

Procedure. The experimenter said "ready" to initiate a trial. A visual warning stimulus, a $7.6\left(5.18^{\circ}\right) \times 12.7 \mathrm{~cm}\left(8.66^{\circ}\right)$ gray rectangle centered on a black background was followed by $1 \mathrm{sec}$ of darkness and then one of the test stimuli, which remained on for $1 \mathrm{sec}$. The keypress was with the index and/or middle finger of the dominant hand, and it stopped the clock. The experimenter said "correct" or "wrong" after each response and also indicated the response time (RT) after each correct response.

The eight (large and small left $\times$ large and small right $\times$ near and far) two-object stimuli occurred once in each block. The eight (large and small $\times$ left and right $\times$ near and far) one-object stimuli also occurred once in each block. In addition, when the target was the large rectangle, there were two additional presentations of both the near and the far small-small stimuli in each block, and when the target was the small rectangle, there were two additional presentations of both the near and the far large-large stimuli in each block. Therefore, there were $\mathbf{2 0}$ trials in each block, and the probability of the target occurring in each block was .50. There were three blocks of trials.

\section{Results}

The analysis of variance (ANOVA) was a 2 (task: identify large rectangle and identify small rectangle) $\times 5$ (object: either large-large or small-small; large-small; smalllarge; either large or small left; and either large or small right) $\times 2$ (distance: near and far) factorial. The entries for the ANOVA were the means of the RTs to each individual stimulus obtained by summing across the three blocks. Only the task factor was between-subject.

Table 1 indicates the mean RTs for the two groups and all stimuli. The only significant outcome was the task $x$ object interaction $[F(4,112)=2.70, p<.05]$. When the target was the large rectangle, the large-small and smalllarge stimuli resulted in an overall mean RT of $423 \mathrm{msec}$, and the large-large, large left, and large right stimuli resulted in an overall mean RT of $425 \mathrm{msec}$. However, when the target was the small rectangle, the large-small and small-large mean RT was $443 \mathrm{msec}$, and the smallsmall, small left, and small right mean RT was $412 \mathrm{msec}$. The 443-msec mean was significantly different from the 412 -msec mean $[F(1,112)=5.50, p<.05]$. Therefore, a large-small slowdown occurred, but only when the small rectangle was the target. In addition, the large-small slowdown for the identification of the small rectangle was significant for the far stimuli: The far large-small and small-large mean RT was $447 \mathrm{msec}$, whereas the far small-small, small left, and small right mean RT was $407 \mathrm{msec}[F(1,112)=12.44, p<.001]$.

The false identifications of a large rectangle as a small rectangle and vice versa were summed across the three blocks for all possible stimuli, and the error percentages are shown in Table 1. An ANOVA was done on the errors that paralleled the one for the RTs, except that the largesmall and small-large stimuli were not levels of the object factor, since errors on these stimuli were not possible. None of the outcomes of the ANOVA were statistically significant.

\section{Discussion}

A large-small slowdown occurred for the identification of the small rectangle but not for that of the large rectangle. This implies that the large rectangle delayed the perception of the small rectangle, but not vice versa. Therefore, the results are consistent with the operating assumption that a large-rectangle superordinate code in-

Table 1

Mean Response Time (RT, in msec) and Percentage of Error (PE) Rate for Each Stimulus in the Identification Experiment

\begin{tabular}{|c|c|c|c|c|c|c|c|c|c|c|c|c|c|c|c|c|}
\hline \multirow[b]{3}{*}{ Target } & \multicolumn{16}{|c|}{ Objects } \\
\hline & \multicolumn{2}{|c|}{ Large-Large } & \multicolumn{2}{|c|}{ Large-Small } & \multicolumn{2}{|c|}{ Small-Large } & \multicolumn{2}{|c|}{ Small-Small } & \multicolumn{2}{|c|}{ Large Left } & \multicolumn{2}{|c|}{ Large Right } & \multicolumn{2}{|c|}{ Small Left } & \multicolumn{2}{|c|}{ Small Right } \\
\hline & RT & $\mathrm{PE}$ & RT & $\mathrm{PE}$ & RT & $\mathrm{PE}$ & RT & $\mathrm{PE}$ & RT & PE & RT & $\mathrm{PE}$ & RT & PE & RT & PE \\
\hline \multicolumn{17}{|c|}{ Near Distance } \\
\hline Large Rectangle & 431 & & 415 & & 423 & & & 2.2 & 445 & & 404 & & & 0.0 & & 4.4 \\
\hline Small Rectangle & & 2.2 & 431 & & 450 & & 433 & & & 6.7 & & 2.2 & 396 & & 420 & \\
\hline \multicolumn{17}{|c|}{ Far Distance } \\
\hline Large Rectangle & 413 & & 424 & & 431 & & & 4.4 & 433 & & 426 & & & 4.4 & & 6.7 \\
\hline Small Rectangle & & 4.4 & 429 & & 464 & & 416 & & & 6.7 & & 2.2 & 410 & & 396 & \\
\hline
\end{tabular}


hibits a small-rectangle superordinate code, thereby delaying the perception of the gestalt of a small rectangle.

Feature explanations. The large-small slowdown for identifying the small rectangle was significant at the far distance. The features of the large and small rectangles were identical over a distance of about $32 \mathrm{~mm}\left(2.18^{\circ}\right)$; that is, $22 \mathrm{~mm}$ (the edge-to-edge distance of the far rectangles) $+5 \mathrm{~mm}$ (the width of the small rectangle) $\times 2$ (two small rectangles). Therefore, features of the large rectangle would have had to have been inhibitory over this distance. However, this distance exceeds the size of the receptive fields of simple and more complex cells in the monkey cortex (Hubel \& Wiesel, 1968), and presumably in the human cortex as well.

The areas inside the large rectangle and outside the small rectangle were both white. Therefore, attributing the large-small slowdown to a difference in the areas of the two rectangles means that these areas involve the enclosing lines as well as the white areas that these lines enclose. Consequently, if the slowdown is attributed to inhibition by the large area feature of the large rectangle, it should be recognized that at least this feature is complex.

Additional explanations. The large and small rectangles are physically different, and this difference-rather than specifically a difference in size-may have slowed down the response to the small-rectangle target. But the large and small rectangles failed to produce the largesmall slowdown when the large rectangle was the target. The same failure also indicates that the physical asymmetry of the large-small and small-large stimuli versus the physical symmetry of the large-large and small-small stimuli could not have been responsible for the slowdown.

Another possibility is that assimilation occurred between the large and small rectangles, thereby delaying the perception of the small rectangle. But this assimilation would have to be asymmetrical to explain why the large-small slowdown did not occur when the large rectangle was the target. In addition, Fuchs (1923/1967) found that reciprocal assimilation occurred between large and small elements. Furthermore, the large and small rectangles are less similar than are the large and large (or small and small) rectangles, and less similar component stimuli seem to reduce assimilation (King, 1988a) and other integrational outcomes (Eriksen \& Collins, 1967; Prinzmetal, 1981).

\section{1-VERSUS-2 EXPERIMENTS}

All five 1-versus-2 experiments employed a countinglike task: Subjects were instructed to respond to two objects but not to one object, or vice versa. Slow two-object responses to the large-small and small-large stimuli would indicate a large-small slowdown. The regular-rectangles experiment, which employed the same stimuli as did the identification experiment, tested the generality of the slowdown and eliminated a response-competition explanation of it. The large(modified)-rectangle experiment moved the vertical lines of the large rectangle closer together. It tested whether replacing the large rectangle gestalt, but keeping the same individual lines, would eliminate the slowdown, contrary to line-feature explanations of the slowdown. The contiguous-rectangles experiment made the two rectangles of the regular-rectangles experiment contiguous. It tested whether replacing two gestalts with one gestalt, but keeping the same areas of the large and small rectangles, would eliminate the slowdown, contrary to the area-feature explanation of the slowdown.

To further test the generality of the large-small slowdown, the circles experiment employed a large solid circle and a small open circle, and the lines experiment replaced the large and small rectangles with lines that differed in orientation. These latter two experiments are of less interest and will be considered only briefly.

\section{Method}

Subjects. For each experiment, one group of 11 subjects was told to respond whenever two objects occurred and otherwise not to respond. A second group of 11 subjects was told to respond whenever one object occurred and otherwise not to respond.

Stimuli. The two-object stimuli of the large(modified)-rectangle experiment are shown in Figure 2. They were identical to those of the regular-rectangles experiment, except that a large(modified) rectangle replaced the large rectangle. To form the large(modified) rectangle, the large rectangle's top and bottom lines were not changed, and its inside and outside lines were moved inward 5 and $10 \mathrm{~mm}$, respectively.

The two-object stimuli of the contiguous-rectangles experiment are shown in Figure 3. They consisted of contiguous rectangles that were otherwise identical to the large and small rectangles of the regular-rectangles experiment. The middle vertical line was always at the center of the tachistoscope card. The one-object stimuli for these three [regular, large(modified), and contiguous] rectangle experiments, as well as for the circles and lines experiments, were the individual objects of the two-object stimuli.
NEAR

LARGE(MODIFIED)LARGE( MODIFIED)

SMALL -

SMALL
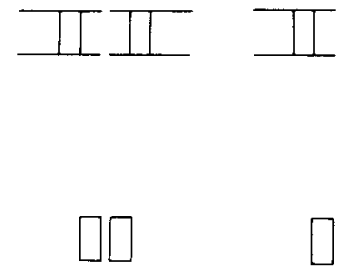

LARGE (MODIFIED)-
SMALL
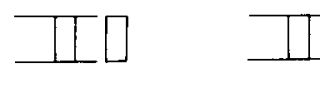

SMALL-

LARGE( MODIFIED)
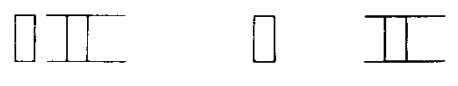

Figure 2. The two-object stimuli for the large(modified)-rectangle experiment. 
LARGE-

LARGE

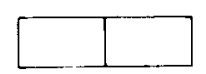

SMALL-

SMALL

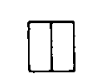

LARGE-

SMALL

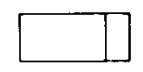

SMALL-

LARGE

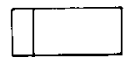

Figure 3. The two-object stimuli for the contiguous-rectangles experiment.

In the circles experiment, a solid black $10-\mathrm{mm}\left(0.68^{\circ}\right)$ diameter circle and an open (unfilled) $4-\mathrm{mm}\left(0.27^{\circ}\right)$ diameter circle replaced the large and small rectangles, respectively. The position and the edge-to-edge distance of these circles on the tachistoscope card were the same as for the rectangles. The line experiment's two-object stimuli are shown in Figure 4. The individual lines were $10 \mathrm{~mm}$ $\left(0.68^{\circ}\right)$ long, and their bottom end points were separated by $2 \mathrm{~mm}$ $\left(0.14^{\circ}\right)$ or $11 \mathrm{~mm}\left(0.75^{\circ}\right)$. The left line was either $45^{\circ}$ to the left of vertical or $10^{\circ}$ to the right of vertical. The right line was either parallel to the left line or rotated $35^{\circ}$ clockwise (see Figure 4 ). The parallel lines correspond to the (identical) large-large and smallsmall pairs, and the nonparallel lines correspond to the (nonidentical) large-small and small-large pairs.

In the regular-rectangles and large(modified)-rectangle experiments, there were 2 [size of left object: large or large(modified) vs. small] $\times 2$ [size of right object: large or large(modified) vs. small] $\times 2$ (distance: near vs. far) $=8$ two-object stimuli, and there were 2 [size: large or large(modified) vs. small] $\times 2$ (distance: near vs. far) $\times 2$ (position: left vs. right) $=8$ one-object stimuli. For the contiguous-rectangles experiment, there was no distance factor; there were only four two-object stimuli and four oneobject stimuli.

Each of the 16 stimuli in the regular-rectangles and large(modified)rectangle experiments occurred once in each block in a random order, whereas each of the eight stimuli of the contiguous-rectangles experiment occurred twice in this manner. For each experiment, there were four blocks in all.

The method for the circles and lines experiments corresponded closely to that of the three rectangle experiments. The method for all five experiments was otherwise identical to that for the identification experiment.

\section{Results}

RTs to two-object stimuli. For the three rectangle experiments, the two-object RTs were analyzed with the two size factors and one distance factor that are indicated above, except that the contiguous-rectangles experiment did not include the distance factor. All factors were withinsubject. The entries for the ANOVA were the means of the two-object RTs to each two-object stimulus obtained by summing across the four blocks. However, the firsttrial RTs of the contiguous-rectangles experiment were disregarded, because they were relatively high.

As shown in Table 2, in the regular-rectangles experiment, the large-small and small-large stimuli resulted in slower two-object responses than did the large-large and small-small stimuli, which parallels the large-small slowdown of the identification experiment. Likewise, the size of left object $\times$ size of right object interaction was significant $[F(1,10)=19.25, p<.01]$. In addition, the near stimuli resulted in a greater large-small slowdown than did the far stimuli $[F(1,10)=5.39, p<.05]$. Nevertheless, the far stimuli resulted in a marginally significant large-small slowdown on their own $[F(1,10)=3.85$, $p<.10$ ]. The only other significant result was that the two-object responses were faster for far stimuli than for near stimuli $[F(1,10)=7.26, p<.05]$.

As shown in Table 3, in the large(modified)-rectangle experiment, the large(modified)-small and small-large (modified) stimuli resulted in slightly faster two-object responses than did the large(modified)-large(modified) and small-small stimuli; there was no large-small-like slowdown. Also, the two-object response was significantly faster for far stimuli than for near stimuli $[F(1,10)=9.30$, $p<.05]$. And the two-object response was faster if
LEFT LINE
LFFT OF
VERTICAL,
SAME
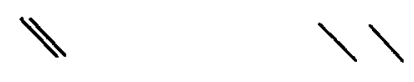

LEFT LINE RIGHT OF VERTICAL, SAME<smiles>C=C</smiles><smiles>CCCC</smiles>

LEFT LINE LFFT OF VERTICAL, DIFFERENT
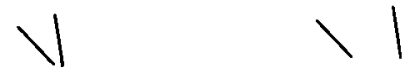

LFFT LINE RICHT OF VERTICAL. DIIFERENI
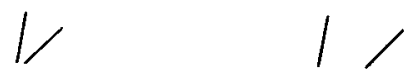

Figure 4. The two-objert stimuli for the lines experiment. 
the left rectangle was large(modified) rather than small $[F(1,10)=6.44, p<.05]$. However, this last result does not relate to the other experiments and hence will not be considered further.

As shown in Table 4, in the contiguous-rectangles experiment, two-object responses were slightly faster to the large-small and small-large stimuli than to the largelarge and small-small stimuli; again there was no largesmall slowdown. The rest of the outcomes were also nonsignificant.

The circles experiment produced a significant largesmall slowdown $[F(1,10)=11.48, p<.01]$, but the lines experiment did not: The mean RT for parallel lines did not differ from the mean RT for nonparallel lines $[F(1,10)=0.11]$. In addition, in both the circles and the lines experiments, the two-object response was faster for far stimuli than for near stimuli $[F(1,10)=0.07$, and $F(1,10)=3.58, p<.10$, respectively $]$.

Finally, to determine if a consistent main effect of distance occurred, the two-object RTs of the four experiments with near and far stimuli were analyzed with a sin- gle ANOVA. The two far objects produced significantly faster responses than did the two near objects $[F(1,40)=$ $15.77, p<.001]$, and the distance $\times$ experiment interaction was nonsignificant $[F(1,40)=1.86]$.

Errors on two-object stimuli. The subjects instructed to respond to one-object stimuli sometimes erred by responding to two-object stimuli. Such false-positive errors were summed across the four blocks, except that firsttrial errors were disregarded for the contiguous-rectangles experiment, to be consistent with the treatment of the RTs for this experiment. The error percentages on two-object stimuli are shown in Tables 2, 3, and 4, and were analyzed in the same fashion as the RTs.

For the regular-rectangles experiment, false-positive errors to the large-small and small-large stimuli were nearly as frequent as those to the large-large and small-small stimuli, and the size of left rectangle $\times$ size of right rectangle interaction was nonsignificant $[F(1,10)=0.06]$. Thus, the large-small slowdown of this experiment (see above) cannot be attributed to a speed-accuracy tradeoff. Errors in the large(modified)-rectangle and contiguous-

Table 2

Mean Response Time (RT, in msec) and Percentage of Error (PE) Rate for Each Stimulus in the Regular-Rectangles Experiment

\begin{tabular}{|c|c|c|c|c|c|c|c|c|c|c|c|c|c|c|c|c|}
\hline \multirow{3}{*}{ Target } & \multicolumn{16}{|c|}{ Objects } \\
\hline & \multicolumn{2}{|c|}{$\underline{\text { Large-Large }}$} & \multicolumn{2}{|c|}{ Large-Small } & \multicolumn{2}{|c|}{ Small-Large } & \multicolumn{2}{|c|}{ Small-Small } & \multicolumn{2}{|c|}{ Large Left } & \multicolumn{2}{|c|}{ Large Right } & \multicolumn{2}{|c|}{ Small Left } & \multicolumn{2}{|c|}{ Small Right } \\
\hline & RT & $\mathrm{PE}$ & RT & $\mathrm{PE}$ & $\mathbf{R T}$ & $\mathrm{PE}$ & RT & PE & $\mathbf{R T}$ & PE & RT & $\mathrm{PE}$ & RT & $\mathrm{PE}$ & $\mathrm{RT}$ & $\mathrm{PE}$ \\
\hline \multicolumn{17}{|c|}{ Near Distance } \\
\hline Two Objects & 345 & & 414 & & 400 & & 377 & & & 2.3 & & 0.0 & & 9.1 & & 9.1 \\
\hline One Object & & 4.5 & & 0.0 & & 2.3 & & 9.1 & 390 & & 390 & & 392 & & 390 & \\
\hline \multicolumn{17}{|c|}{ Far Distance } \\
\hline Two Objects & 350 & & 355 & & 378 & & 348 & & & 6.8 & & 6.8 & & 6.8 & & 0.0 \\
\hline One Object & & 2.3 & & 9.1 & & 4.5 & & 2.3 & 399 & & 396 & & 381 & & 389 & \\
\hline
\end{tabular}

Table 3

Mean Response Time (RT, in msec) and Percentage of Error (PE) Rate for Each Stimulus in the Large(Modified)-Rectangle Experiment Objects

\begin{tabular}{|c|c|c|c|c|c|c|c|c|c|c|c|c|c|c|c|c|}
\hline \multirow[b]{2}{*}{ Target } & \multicolumn{2}{|c|}{$\begin{array}{c}\text { Large } \\
\text { (modified)- } \\
\text { Large } \\
\text { (modified) }\end{array}$} & \multicolumn{2}{|c|}{$\begin{array}{c}\text { Large } \\
\text { (modified)- } \\
\text { Small }\end{array}$} & \multicolumn{2}{|c|}{$\begin{array}{c}\text { Small-Large } \\
\text { (modified) }\end{array}$} & \multicolumn{2}{|c|}{ Small-Small } & \multicolumn{2}{|c|}{$\begin{array}{c}\text { Large } \\
\text { (modified) } \\
\text { Left }\end{array}$} & \multicolumn{2}{|c|}{$\begin{array}{c}\text { Large } \\
\text { (modified) } \\
\text { Right }\end{array}$} & \multicolumn{2}{|c|}{ Small Left } & \multicolumn{2}{|c|}{ Small Right } \\
\hline & RT & $\mathrm{PE}$ & RT & $\mathrm{PE}$ & RT & $\mathrm{PE}$ & RT & $\mathrm{PE}$ & RT & $\mathrm{PE}$ & RT & $\mathrm{PE}$ & RT & PE & RT & PE \\
\hline \multicolumn{17}{|c|}{ Near Distance } \\
\hline Two Objects & 379 & & 403 & & 409 & & 432 & & & 2.3 & & 4.5 & & 0.0 & & 2.3 \\
\hline One Object & & 0.0 & & 4.5 & & 0.0 & & 15.9 & 407 & & 414 & & 376 & & 366 & \\
\hline \multicolumn{17}{|c|}{ Far Distance } \\
\hline Two Objects & 371 & & 367 & & 376 & & 379 & & & 9.1 & & 6.8 & & 4.5 & & 4.5 \\
\hline One Object & & 2.3 & & 2.3 & & 2.3 & & 4.5 & 442 & & 412 & & 389 & & 365 & \\
\hline
\end{tabular}

Table 4

Mean Response Time (RT, in msec) and Percentage of Error (PE) Rate for Each Stimulus in the Contiguous-Rectangles Experiment Objects

\begin{tabular}{|c|c|c|c|c|c|c|c|c|c|c|c|c|c|c|c|c|}
\hline \multirow[b]{2}{*}{ Target } & \multicolumn{2}{|c|}{ Large-Large } & \multicolumn{2}{|c|}{ Large-Small } & \multicolumn{2}{|c|}{ Small-Large } & \multicolumn{2}{|c|}{ Small-Small } & \multicolumn{2}{|c|}{ Large Left } & \multicolumn{2}{|c|}{ Large Right } & \multicolumn{2}{|c|}{ Small Left } & \multicolumn{2}{|c|}{ Small Right } \\
\hline & $\overline{R T}$ & $\mathrm{PE}$ & $\mathrm{RT}$ & $\mathrm{PE}$ & $\mathrm{RT}$ & $\mathrm{PE}$ & $\mathrm{RT}$ & PE & RT & $\mathrm{PE}$ & RT & $\mathrm{PE}$ & RT & PE & $\mathrm{RT}$ & $\mathrm{PE}$ \\
\hline Two Objects & 379 & & 377 & & 374 & & 378 & & & 2.3 & & 0.0 & & 2.3 & & $\overline{2.3}$ \\
\hline One Object & & 1.1 & & 0.0 & & 3.4 & & 5.7 & 413 & & 407 & & 375 & & 381 & \\
\hline
\end{tabular}


rectangles experiments did not hint at a difficulty with large-small and small-large stimuli, in accord with the RT results of these experiments. In addition, the size of left rectangle $\times$ size of right rectangle interactions were nonsignificant $[F(1,10)=1.75$ for the large(modified)rectangle experiment, and $F(1,10)=1.32$ for the contiguous-rectangles experiment]. In the circles experiment, errors on the large-small and small-large stimuli exceeded those on the large-large and small-small stimuli $[F(1,10)=12.28, p<.01]$, in accord with this experiment's large-small slowdown. In the lines experiment, errors on the parallel lines exceeded, but not significantly so, errors on the nonparallel lines $[F(1,10)=2.22$, $p>.10]$.

Errors on far stimuli exceeded those on near stimuli in the regular-rectangles $[F(1,10)=0.06]$ and circles $[F(1,10)=0.45]$ experiments, but the reverse occurred in the large(modified)-rectangle $[F(1,10)=1.70]$ and lines $[F(1,10)=3.75, p<.10]$ experiments. Therefore, the faster two-object responses to the far stimuli cannot have been due to a speed-accuracy tradeoff.

RTs to one-object stimuli. For the three rectangle experiments, the one-object RTs were analyzed with the size, distance, and position factors that were previously indicated, except that the contiguous-rectangles experiment did not include the distance factor. All factors were within-subject. The RTs were summed as for the twoobject RTs. Tables 2, 3, and 4 contain the mean RT for each one-object stimulus of these three experiments.

In the regular-rectangles experiment, the one-object response was slower to the large rectangle than to the small rectangle, but not significantly so $[F(1,10)=1.12]$. However, in the large(modified)-rectangle and contiguousrectangles experiments, the large(modified) and large rectangles did produce significantly slower one-object responses than did the small rectangle $[F(1,10)=14.66$, $p<.01$, and $F(1,10)=15.91, p<.01$, respectively]. There were no other statistically significant outcomes. In the circles experiment, by contrast, the large circle produced faster one-object responses than did the small circle $[F(1,10)=7.04, p<.05]$

Errors on one-object stimuli. The subjects instructed to respond to two-object stimuli sometimes erred by responding to one-object stimuli. Such false-positive errors were summed in the same way as the errors on two-object stimuli. The error percentages are shown in Tables 2, 3, and 4 , and were analyzed in the same fashion as the RTs.

Slightly fewer errors were made on the large single object than on the small single object in the regularrectangles $[F(1,10)=0.41]$ and contiguous-rectangles $[F(1,10)=0.13]$ experiments, whereas the reverse occurred in the large(modified)-rectangle $[F(1,10)=1.54]$ and circles $[F(1,10)=0.05]$ experiments. Therefore, the significant effects of size on one-object RTs cannot have been due to speed-accuracy tradeoffs.

Comparisons between one-object and two-object RTs and errors. For the regular-rectangles and large (modified)-rectangle experiments, the one- and two-object
RTs were compared with 2 (response: one object vs. two objects) $\times 2$ [size: large or large(modified) vs. small] $\times$ 2 (distance: near vs. far) ANOVAs. For the contiguousrectangles experiment, there was no distance factor. The response factor was between-subject; the rest were withinsubject. The ANOVAs were restricted to the large-large or large(modified)-large(modified) and the small-small two-object stimuli, because only these stimuli corresponded to the large or large(modified) and small one-object stimuli. The entries to the ANOVA for the one-object responses were collapsed across the left and right positions.

For the three rectangle experiments, the two-object responses were faster, but not significantly so, than the oneobject responses $[F(1,20)=3.98, p<.10, F(1,20)=$ 0.04 , and $F(1,20)=0.71$, respectively]. The two-object responses were also faster than the one-object responses in the circles and lines experiments $[F(1,20)=4.74, p$ $<.05$, and $F(1,20)=0.33$, respectively]. No error comparisons were significant ( $F \leq 1.38$ in all cases).

\section{Discussion}

Superordinate-is-causal theory. Only the regularrectangles and circles experiments resulted in the largesmall slowdown. According to phenomenal evidence, both the large-small and the small-large stimuli of these experiments result in two gestalts, one of a large object and the second of a small object. Therefore, according to the present theory, in both of these experiments the largeobject superordinate code inhibited the small-object superordinate code, thereby delaying the perception of the small-object gestalt and resulting in the slowdown, just as in the identification experiment.

The large(modified) rectangle does not produce a gestalt of a large object. Therefore, the present theory attributes the absence of the large-small slowdown in the large (modified)-rectangle experiment to the inability of the large(modified)-rectangle superordinate code to inhibit the small-rectangle superordinate code. The absence of the analogous slowdown in the lines experiment is explained in the same way.

In additional tests, using a 7-point scale, single large, small, and large(modified) rectangles were rated on the extent to which they were apprehended as either one or two gestalts. The large(modified) rectangle was judged to be a weaker single gestalt than were either the large rectangle or the small rectangle (sign test, $p<.01$, twotailed, for both comparisons). Thus, the present theory also assumes that the large(modified)-rectangle superordinate code was sufficiently weak that it did not inhibit the small-rectangle superordinate code, and hence no slowdown occurred.

Phenomenally, the two contiguous rectangles produce a strong single gestalt. Therefore, the present theory posits that the two contiguous rectangles ought to produce a single superordinate code. Consequently, two superordinate codes did not occur, and hence did not interact to produce the large-small slowdown. This accounts 
for the absence of the large-small slowdown in the contiguous-rectangles experiment.

Response competition. Irrelevant letters that signal a competing response interfere with the response to a target letter, especially when they are relatively large (Eriksen \& Schultz, 1979). A nonresponse may function like a competing response. If so, in the identification experiment, when the small rectangle was the target, the large rectangle of the large-small and small-large stimuli may have primed a strong competing nonresponse. In addition, when the large rectangle was the target, the small rectangle of the same stimuli may have primed only a weak competing nonresponse. Therefore, response competition accounts for the large-small slowdown of the identification experiment.

In the regular-rectangles experiment, when the task was to respond to two objects, single objects were assigned to a nonresponse. Therefore, each object of a two-object stimulus may have primed a competing nonresponse. In addition, the large rectangles of the large-small and smalllarge stimuli may have primed the competing nonresponse more than did the small rectangles of the small-small stimuli. This would account for the slower two-object responses to the large-small and small-large stimuli than to the small-small stimuli. If so, however, then each large rectangle of the large-large stimuli also should have strongly primed the competing nonresponse, and the largelarge stimuli should have produced slow two-object responses, which was not the case. This casts doubt on the response-competition explanation, although the large size of the large-large stimuli may explain their low RTs. The response-competition explanation also fails to account for the difference in results between the regular-rectangles and contiguous-rectangles experiments.

Feature explanations. The large rectangle is greater in area, as well as in line length, than the small rectangle. In addition, its lines contrast more with the background than do the small rectangle's lines. These features of the large rectangle may interfere with the perception of the small rectangle.

The contiguous-rectangles experiment eliminated the feature factor, however. This is because the large and small rectangles of this experiment were identical in size, shape, height-to-width ratio, other relations between lines, and type of adjacency to those of the regular-rectangles experiment, and yet the large-small slowdown did not occur. Furthermore, sheer closeness or "nearness per se" did not eliminate the slowdown, because in the regularrectangles experiment, the near rectangles produced a greater slowdown than did the far rectangles.

The large(modified) rectangle contains the same lines and much the same line-line relations as does the large rectangle. However, the large(modified)-rectangle experiment did not produce the large-small slowdown. This, too, counters feature explanations of the large-small slowdown. Feature theory seemingly has run out of features that can readily account for the slowdown.

The far-apart stimuli resulted in at least a marginally significant large-small slowdown in the regular-rectangles and circles experiments. Therefore, any difference in features between the large rectangle and the small rectangle would probably have had to have been influential over the 32-mm $\left(2.18^{\circ}\right)$ distance at which the far large rectangle and the far small rectangle begin to differ, which is questionable (see the identification experiment discussion).

Additional explanations. The 1-versus- 2 results argue against the same-different, size asymmetry, and assimilation explanations of the large-small slowdown in the same way that the identification results do. For example, the large and small contiguous rectangles, the large(modified) and small rectangles, and the nonparallel lines were not identical, but they did not result in the slowdown. Therefore, the nonidentity of the large and small rectangles of the regular-rectangles experiment should not have been responsible for its large-small slowdown.

The contiguous-rectangles experiment argues against the assimilation explanation of the large-small slowdown in additional ways. The contiguous rectangles produce a stronger single gestalt than do the regular rectangles, and are also closer to each other than are the regular rectangles. In addition, strong single gestalts are associated with extensive assimilation (Fuchs, 1923/1967; King, 1988a, 1988b, 1988c), and proximal stimuli result in extensive assimilation (King, 1988a). Therefore, the contiguous rectangles should have resulted in more assimilation, and hence, if assimilation were a factor, a greater large-small slowdown. However, the contiguous rectangles produced no slowdown at all.

Distance and two-object RTs. Although the near stimuli resulted in a greater large-small slowdown than did the far stimuli in the regular-rectangles experiment, they did not in the identification and circles experiments $(F<0.23)$. Therefore, it seems difficult to interpret the regular-rectangles result.

Two far objects produced a faster two-object response than did two near objects. This main effect of distance replicates earlier 1-versus-2 results (King, 1990). He also obtained a distance $\times$ gestalt strength interaction: If two objects produced one gestalt when near, the two objects were responded to slowly when near but quickly when far; whereas if each of two objects produced its own gestalt when near (i.e., two gestalts in all), the two objects were responded to quickly at either distance. The near-to-far increase in distance also markedly affected gestalt strength, but only for the two objects that produced one gestalt when near: each of these objects produced its own gestalt when far. Similarity and other factors were controlled. The implication is that both the King (1990) results and the present main effects of distance occurred because the increase in distance enhanced the extent to which each object produced its own gestalt.

The slow one-object responses to the large(modified) rectangle. The large(modified) rectangle resulted in slower one-object responses than did the small rectangle of the same experiment. This result contrasts with evidence that large objects are usually identified more quickly than small objects (Eriksen \& Schultz, 1979; Grice et al., 1983; 
Navon, 1977; Schultz \& Eriksen, 1978). The vertical lines of the large(modified) rectangle are closer together than those of the large rectangle. Therefore, the result is also surprising when considered in terms of acuity.

The large(modified) rectangle was rated as a weaker single gestalt than either the large rectangle or the small rectangle (see above). Therefore, a single object produced a weaker single gestalt and slower one-object responses, which is consistent with evidence (King, 1990) that two objects produced a weaker single gestalt and faster twoobject responses.

The slow one-object responses to the large rectangle. In the contiguous-rectangles experiment, the one-object response was slower for the large rectangle than for the small rectangle, which is also surprising. Contiguous rectangles tend to result in a physically continuous stimulus with a large total size: Outside perimeter length and the area this perimeter encloses tend to be large. Likewise, single rectangles tend to result in a small total size. Therefore, in the contiguous-rectangles experiment, two-object responses tended to be assigned to large total-size stimuli, and one-object responses to small total-size stimuli. Consequently, one might predict that the two-object responses to large total-size stimuli and the one-object responses to small total-size stimuli would both be fast. The total size of a one-object large rectangle exceeds that of a one-object small rectangle. The slow one-object response to the large rectangle follows.

But this account incorrectly predicts faster two-object responses as the total size of the two-object stimuli increases in the contiguous-rectangles experiment. Perhaps the requirement to respond to two objects made the subjects attend to each of the objects, thereby blocking an effect of total size.

\section{CONCLUSIONS}

Superordinate-is-causal theory accounts for the present large-small slowdown results. This theory is additionally supported by the disconfirmations of the feature theory predictions and of several other predictions as well.

Nevertheless, every change in a stimulus introduces new features. Thus, one might posit that the long horizontal lines that result by making two rectangles contiguous underlay the absence of the large-small slowdown in the contiguous-rectangles experiment. However, the lengths of the horizontal lines of the large rectangle and of the large(modified) rectangle are equal, and yet the slowdown occurred in the regular-rectangles experiment but not the large(modified)-rectangle experiment. This suggests that horizontal line length is not critical. Feature theory should not be used so flexibly that it becomes unfalsifiable.

In conclusion, the present research suggests that a superordinate code was the direct (immediate) cause of both the gestalt of a large rectangle and the associated perceptual outcome of a delay in the perception of the gestalt of a small rectangle. Extrapolating, this research supports the possibility that superordinate codes are the direct cause of the numerous other gestalts and associated perceptual outcomes that were considered in the introduction.

\section{REFERENCES}

Banks, W. P., \& Prinzmetal, W. (1976). Configurational effects in visual information processing. Perception \& Psychophysics, 19, 361-367

Brigell, M., UhLARIK, J. (1979). The relational determination of length illusions and length aftereffects. Perception, 8, 187-197.

Bundesen, C., \& Pedersen, L. F. (1983). Color segregation and visual search. Perception \& Psychophysics, 33, 487-493.

Ceraso, J., Powers, T., Moncrief, J. (1988, November). Target detection with homogeneous and heterogeneous distractors. Paper presented at the annual meeting of the Psychonomic Society, Chicago, IL.

ERIKsEN, C. W., Collins, J. F. (1967). Some temporal characteristics of visual pattern perception. Journal of Experimental Psychology, 74, 476-484.

EriKSEN, C. W., Schultz, D. W. (1979). Information processing in visual search: A continuous flow conception and experimental results. Perception \& Psychophysics, 25, 249-263.

FrYKLUND, I. (1975). Effects of cued-set spatial arrangement and targetbackground similarity in the partial-report paradigm. Perception \& Psychophysics, 17, 375-386.

FucHs, W. (1967). The influence of form on the assimilation of colors. In W. D. Ellis (Ed. and Trans.), A source book of gestalt psychology (pp. 95-103). New York: Humanities Press. (Original work published 1923)

GARNER, W. R., \& Felfoldy, G. L. (1970). Integrality of stimulus dimensions in various types of information processing. Cognitive Psychology, 1, 225-241.

GAYLORD, S. (1984). Effects of inter-stimulus distances on the formation of category boundaries. Unpublished doctoral dissertation, Duke University, Durham, NC.

GiBson, J. J. (1933). Adaptation, after-effect and contrast in the perception of curved lines. Journal of Experimental Psychology, 41, 1-31.

GoGEL, W. C. (1977). The metric of visual space. In W. Epstein (Ed.), Stability and constancy in visual perception: Mechanisms and processes (pp. 129-181). New York: Wiley.

Gravetter, F. Lockhead, G. R. (1973). Criterial range as a frame of reference for stimulus judgment. Psychological Review, 80, 203-216.

Grice, G. R., Canham, L., Boroughs, J. M. (1983). Forest before trees? It depends where you look. Perception \& Psychophysics, 33, 121-128.

Hubel, D. H., \& Wiesel, T. N. (1968). Receptive fields and functional architecture of monkey striate cortex. Journal of Physiology, 195, 215-243.

IKeDA, H., ObonaI, T. (1955). The studies in figural aftereffects: IV. The contrast-confluence illusion of concentric circles and the figural after-effect. Japanese Psychological Research, 2, 17-23.

Kahneman, D., Henik, A. (1981). Perceptual organization and attention. In M. Kubovy \& J. R. Pomerantz (Eds.), Perceptual organization (pp. 181-211). Hillsdale, NJ: Erlbaum.

KING, D. L. (1988a). Assimilation is due to one perceived whole and contrast is due to two perceived wholes. New Ideas in Psychology, 6, 277-288.

KING, D. L. (1988b). The Kanizsa and Lockhead commentaries. New Ideas in Psychology, 6, 301-305.

KING, D. L. (1988c). Strong phenomenal wholes are associated with fast "same" and slow "different" responses and superior overall performance. Perception \& Psychophysics, 43, 485-493.

KING, D. L. (1990). Gestalts are more closely associated with performance on a discrimination task than are component stimuli. American Journal of Psychology, 103, 37-52. 
KING, D. L., \& ATEF-VAHID, M-K. (1986). Two extensions of the anchor-range effect. Perception \& Psychophysics, 39, 96-104.

Lockhead, G. R. (1972). Processing dimensional stimuli: A note. Psychological Review, 79, 410-419.

LockHEAD, G. R. (1988). Assimilation and contrast: Two processes or one? New Ideas in Psychology, 6, 293-299.

NAvoN, D. (1977). Forest before trees: The precedence of global features in visual perception. Cognitive Psychology, 9, 353-383.

Piaget, J., \& Lambercier, M. (1944). Recherches sur le developpement des perceptions: V. Essai sur un effet d' "Einstellung'" survenant au cours de perceptions visuelles, successives (effet Usnadze). $A r$ chives de Psychologie, Geneve, 30, 139-196.

POLLACK, I. (1952). The information of elementary auditory displays. Journal of the Acoustical Society of America, 24, 745-749.

Pollack, R. H. (1964). Simultaneous and successive presentation of elements of the Mueller-Lyer figure and chronological age. Perceptual \& Motor Skills, 19, 303-310.

Pollatsek, A., \& Digman, L. (1977). Dependent spatial channels in visual processing. Cognitive Psychology, 9, 326-352.
Prinzmetal, W. (1981). Principles of feature integration in visual perception. Perception \& Psychophysics, 30, 330-340.

Prinzmetal, W., Banks, W. P. (1977). Good continuation affects visual detection. Perception \& Psychophysics, 21, 389-395.

Prinzmetal, W., \& Keysar, B. (1989). Functional theory of illusory conjunctions and neon colors. Journal of Experimental Psychology: General, 118, 165-190.

Sagara, M., \& Oyama, T. (1957). Experimental studies on figural aftereffects in Japan. Psychological Bulletin, 54, 327-338.

Schultz, D. W., \& ERIKSEN, C. W. (1978). Stimulus size and acuity in information processing. Bulletin of the Psychonomic Society, 12, 397-399.

WELCH, R. (1978). Perceptual modification: Adapting to altered sensory environments. New York: Academic Press.

WELCH, R., WARREN, D. H. (1980). Immediate perceptual response to intersensory discrepancy. Psychological Bulletin, 88, 638-667.

(Manuscript received October 3, 1988; revision accepted for publication October $17,1989$. 